Canad. Math. Bull. Vol. 20 (3), 1977

\title{
ABELIAN THEOREMS FOR HARDY TRANSFORMATIONS
}

\author{
BY \\ R. S. PATHAK AND J. N. PANDEY
}

ABSTRACT. Initial and final value theorems for Hardy transformations $\int_{0}^{\infty} f(x) C_{\nu}(x y) d x$ and $\int_{0}^{\infty} f(x) F_{v}(x y) d x$ of a suitably chosen function $f(x)$ under a certain set of conditions on $\nu$ and $p$ where

$$
C_{\nu}(x)=\cos p \pi J \nu(x)+\sin p \pi Y_{\nu}(x)
$$

$J_{\nu}(x)$ and $Y_{\nu}(x)$ being Bessel functions of the first and second kind, and

$$
F_{\nu}(x)=2^{2-\nu-2 p} s_{\nu+2 p-1, \nu}(x) /\{\Gamma(p) \Gamma(\nu+p)\}
$$

$s_{u, v}(x)$ being Lommel's function, are proved.

1. Introduction. Applications of Abelian theorems in solving boundary value problems are well known. Abelian theorems for Laplace transforms are given by Widder [8] and that for the Hankel transform are given by Zemanian [9]. Abelian theorems for $\boldsymbol{Y}$ - and $\boldsymbol{H}$-transforms are not available in Literature. In the following we give Abelian theorems for the Hardy transforms which incorporate $Y$ - and $H$ - transforms as special cases.

TheOREM 1. Let $3 / 2<\eta<2-\left|\operatorname{Re}^{\nu}\right|$ where $\nu$ is complex. Let $f(x)$ be a measurable function on the interval $(0, \infty)$ such that $x^{\eta} f(x)$ is Lebesgue integrable on every interval of the form $(x, \infty), X>0$.

Assume that

$$
\lim _{x \rightarrow 0+} x^{\eta} f(x)=\lambda,
$$

where $\lambda$ is complex in general and define the Hardy transformation $F(y)$ of $f(x)$ by

$$
F(y)=\int_{0}^{\infty} f(x) \times C_{\nu}(x y) d x
$$

where $C_{v}(x)$ is the function defined by (1).

Then

$$
\lim _{y \rightarrow \infty} y^{2-\eta} F(y)=\lambda G(\nu, \eta)
$$

Received by the editors June 7, 1976. 
where

$$
G(\nu, \eta)=\frac{\cos (p \pi) \Gamma\left(\frac{2+\nu-\eta}{2}\right)}{2^{\eta-1} \Gamma\left(\frac{\nu+\eta}{2}\right)} \frac{\sin p \pi \cot \left[\frac{(\nu+\eta) \pi}{2}\right] \Gamma\left(1+\frac{\nu-\eta}{2}\right)}{2^{\eta-1} \Gamma\left(\frac{\nu+\eta}{2}\right)}
$$

p being a complex number.

Proof. From (3), $f(x)=0\left[x^{-\eta}\right], x \rightarrow 0+$. Since,

$$
C_{\nu}(x)=\begin{array}{ll}
0\left[x^{-|\nu|}\right], & x \rightarrow 0+ \\
0\left[x^{-1 / 2}\right], & x \rightarrow \infty
\end{array}
$$

the Hardy transform $F(y)$ of $f(x)$ exists.

Now form [2; pp. 326, 329]

$$
\int_{0}^{\infty} t^{1-\eta} C_{\nu}(t) d t=G(\nu, \eta) ; \quad 1<\eta<2-\left|\operatorname{Re}^{\nu}\right| .
$$

using the transformation $t=x y, y>o$ in (6) we have

$$
\int_{0}^{\infty} y^{2-\eta} x^{1-\eta} C_{\nu}(x y) d x=G(\nu, \eta)
$$

Therefore, in view of (4) and (7) we have

(9) $\left|y^{2-\eta} F(y)-\lambda G(\nu, \eta)\right| \leq y \int_{0}^{\infty}\left|x^{\eta} f(x)-\lambda\right|\left|(x y)^{1-\eta} C_{\nu}(x y)\right| d x$

$$
\begin{aligned}
& =y \int_{0}^{\delta}\left|x^{\eta} f(x)-\lambda\right|\left|(x y)^{1-\eta} C_{\nu}(x y)\right| d x \\
& +y \int_{\delta}^{\infty}\left|x^{\eta} f(x)-\lambda\right|\left|(x y)^{1-\eta} C_{\nu}(x y)\right| d x, \delta>0 \\
& \leqq \sup _{0<t \leq \delta}\left|t^{\eta} f(t)-\lambda\right| \int_{0}^{\delta}\left|x^{1-\eta} C_{\nu}(x)\right| d x \\
& +y^{3 / 2-\eta} \int_{\delta}^{\infty}\left|x^{1 / 2} f(x)-\lambda x^{-\eta+1 / 2}\right|\left|\sqrt{ }(x y) C_{\nu}(x y)\right| d x
\end{aligned}
$$

Since $\int_{0}^{\infty}\left|x^{1-\eta} C_{\nu}(x)\right| d x$ is convergent in view of (3) for $\varepsilon>0$ we can choose a positive $\delta$ such that

$$
\left|t^{n} f(t)-\lambda\right|<\frac{\varepsilon}{2 \int_{0}^{\infty}\left|x^{1-\eta} C_{\nu}(x)\right| d x}
$$

Fix $\delta$ this way. Therefore in view of (8)

(10) $\left|y^{2-\eta} F(y)-\lambda G(\nu, \eta)\right|<\frac{\varepsilon}{2}+y^{(3 / 2)-\eta} \int_{0}^{\infty}\left|x^{1 / 2} f(x)-\lambda x^{-\eta+1 / 2}\right|\left|\sqrt{ } x y C_{\nu}(x y)\right| d x$. 
Let

$$
\sup _{x>0}\left|\sqrt{ } x C_{\nu}(x)\right|=K
$$

and

$$
\int_{0}^{\infty}\left|x^{1 / 2} f(x)-\lambda x^{-\eta+1 / 2}\right| d x=C
$$

Then, from (9)

$$
\left|y^{2-\eta} F(y)-\lambda G(\nu, \eta)\right|<\frac{\varepsilon}{2}+y^{3 / 2-\eta} K C .
$$

We can now choose $N>0$ sufficiently large such that

$$
y^{3 / 2-\eta} K C<\frac{\varepsilon}{2} \text { for all } y>N .
$$

Now from (10) and (11) we have

$$
\left|y^{2-\eta} F(y)-\lambda G(\nu, \eta)\right|<\varepsilon \text { for all } y>N .
$$

Since $\varepsilon$ is arbitrary our result is proved.

THEOREM 2. Let $\nu$ and $p$ be complex numbers and $\eta$ a real number satisfying $3 / 2<\eta<2-\left|\operatorname{Re}^{v}\right|$. Assume that $f(x)$ is a measurable function in $(0, \infty)$ such that $x^{1-\left|\mathrm{Re}^{\mathrm{v}}\right|} f(x)$ is Lebesgue integrable on every interval of the form $0<x<X(X<\infty)$ and that there exists a complex number $\lambda$ such that

$$
\lim _{x \rightarrow \infty} x^{\eta} f(x)=\lambda .
$$

Then with $F(y)$ and $G(\nu, \eta)$ as defined by (4) and (6) respectively

$$
\lim _{y \rightarrow 0+} y^{2-\eta} F(y)=\lambda G(\nu, \eta) .
$$

Proof. Since $C_{\nu}(x)=0\left(x^{-|\nu|}\right)$ as $x \rightarrow 0+$ and $C_{\nu}(x)=0\left(x^{-1 / 2}\right)$ as $x \rightarrow \infty$ our conditions on $f(x)$ insure that the transform $F(y)$ of $f(x)$ as defined by (4) exists for $y>0$.

Now,

$$
\begin{aligned}
\left|y^{2-\eta} F(y)-\lambda G(\nu, \eta)\right| \leq y \int_{0}^{\infty}\left|x^{\eta} f(x)-\lambda\right|\left|(x y)^{1-\eta} C_{\nu}(x y)\right| d x \\
\quad \leq y \int_{0}^{x}\left|x^{\eta} f(x)-\lambda\right|\left|(x y)^{1-\eta} C_{\nu}(x y)\right| d x \\
+y \int_{x}^{\infty}\left|x^{\eta} f(x)-\lambda\right|\left|(x y)^{1-\eta} C_{\nu}(x y)\right| d x
\end{aligned}
$$


Exploiting (13) for $\varepsilon>0$ we can find $X>0$ such that

Therefore,

$$
\left|f(x) x^{\eta}-\lambda\right|<\frac{\varepsilon}{\int_{0}^{\infty} t^{1-\eta}\left|C_{\nu}(t)\right| d t} \text { for all } x>X
$$

$$
\left|y^{2-\eta} F(y)-\lambda G(\nu, \eta)\right|<y \int_{0}^{x}\left|x^{\eta} f(x)-\lambda\right|(x y)^{1-\eta} C_{\nu}(x y) d x+\varepsilon
$$

or

$$
\left|y^{2-\eta} F(y)-\lambda G(\nu, \eta)\right|<\varepsilon+y^{3 / 2-\eta} \int_{0}^{X}\left|f(x)-\lambda x^{-\eta}\right| \mid C_{\nu}(x y) \sqrt{ } x y \sqrt{ } x d x
$$

There exists a positive number $A_{\nu}$ such that

$$
\left|\sqrt{ } t C_{\nu}(t)\right| \leq A_{\nu} t^{-|\operatorname{Re}|+1 / 2} \text { for all } t>0 .
$$

Therefore exploiting (14) and (15) we have

$$
\begin{aligned}
\left|y^{2-\eta} F(y)-\lambda G(\nu, \eta)\right| & <\varepsilon+A_{\nu} y^{2-\eta-|\operatorname{Re}|} \int_{0}^{x}\left|f(x)-\lambda x^{-\eta}\right| x^{-\left|\operatorname{Re}^{\nu}\right|+1} d x \\
& <\varepsilon+A_{\nu} y^{2-\eta-\left|\operatorname{Re}^{\nu}\right|} \int_{0}^{x}\left|f(x)-\lambda x^{-\eta}\right| x^{-\left|\operatorname{Re}^{\nu}\right|+1} d x .
\end{aligned}
$$

Letting $y \rightarrow 0+$ we have

$$
\varlimsup_{y \rightarrow 0+}\left|y^{2-\eta} F(y)-\lambda G(\nu, \eta)\right| \leqslant \varepsilon .
$$

Since $\varepsilon$ is arbitrary our lemma is proved.

TheOREM 3. Let $\sigma+2<\eta<\operatorname{Re}(\nu+2 p+2)$ where $\sigma=\max \left(-\frac{1}{2}, \operatorname{Re}(\nu+2 p-2)\right)$ $\nu$ and $p$ being complex numbers. Let $f(x)$ be a measurable function on $0<x<\infty$ such that $x^{\sigma+1} f(x)$ is Lebesgue integrable on every interval of the form $X<x<\infty$ $(X>0)$ and that there exists a complex number such that $\lim _{x \rightarrow 0+} x^{\eta} f(x)=\lambda$.

Let the $F_{\nu}$-transform of $f(x)$ be defined by

$$
F(y)=\int_{0}^{\infty} f(x) F_{\nu}(x y) x d x \text { for each } y>0,
$$

where

$$
F_{\nu}(x)=\frac{2^{2-\nu-2 p} s_{\nu+2 p-1, \nu}(x)}{\{\Gamma(p) \Gamma(\nu+p)\}}
$$

$s_{\mu, \nu}(x)$ being Lommel's function. Then

$$
\lim _{y \rightarrow \infty} y^{2-\eta} F(y)=\lambda H(\nu, \eta)
$$


where

$$
H(\nu, \eta)=\frac{\Gamma\left(\frac{\eta-\nu-2 p}{2}\right) \Gamma\left(1-\frac{\eta-\nu-2 p}{2}\right)}{2^{\eta-1} \Gamma\left(\frac{\eta-\nu}{2}\right) \Gamma\left(\frac{\eta+\nu}{2}\right)}, \frac{1}{2}<\eta<2+\operatorname{Re}(\nu+2 p) .
$$

Proof. The proof follows quite readily by using the fact that

$$
\int_{0}^{\infty} t^{1-\eta} F_{\nu}(t) d t=H(\nu, \eta) ; \quad \eta<2+\operatorname{Re}(\nu+2 p),
$$

and the technique used in the proof of Theorem 1 .

Theorem 4. Let $\sigma+2<\eta<\operatorname{Re}(\nu+2 p)+2$ where $\sigma=\max \left(-\frac{1}{2},-\operatorname{Re}(\nu+\right.$ $2 p+2)$ ) $\nu$ and $p$ being complex constant. Let $f(x)$ be a measurable function on $0<x<\infty$ such that $x^{\nu+2 p+1} f(x)$ is Lebesgue integrable over any interval of the form $0<x<X(X<\infty)$ and that $\lim _{x \rightarrow \infty} x^{\eta} f(x)=\lambda, \lambda$ being $a$ complex number in general.

Then with $F(y)$ and $H(\nu, \eta)$ as defined by (16) and (17) respectively,

$$
\lim _{y \rightarrow 0+} y^{2-\eta} F(y)=\lambda H(\nu, \eta) \text {. }
$$

Proof. The proof follows quite readily by using the fact that

$$
\int_{0}^{\infty} t^{1-\eta} F_{\nu}(t) d t=H(\nu, \eta), \eta<2+\operatorname{Re}(\nu+2 p)
$$

and the technique used in the proof of Theorem 2.

\section{REFERENCES}

1. R. G. Cooke, The inversion formulae of Hardy and Titchmarsh. Proc. London Math. Soc. 24 (1925), 381-420.

2. A. Erdély, (Editor). Tables of integral transforms, Vol. I (McGraw-Hill Book Co., Inc., New York, 1954).

3. A. Erdély, (Editor). Tables of integral transforms, Vol. II (McGraw-Hill Book Co., Inc., New York, 1954).

4. G. H. Hardy, Some formulae in the theory of Bessel functions. Proc. London Math. Soc. 23 (1925), lxi-lxiii.

5. R. S. Pathak, and J. N. Pandey, A distributional Hardy transformation. Proc. Camb. Phil. Soc. 76 (1974), 247-262.

6. R. S. Pathak, and J. N. Pandey, A distributional Hardy transformation II. Submitted for publication.

7. G. N. Watson, A treatise on the theory of Bessel functions (Cambridge University Press, second edn., 1962).

8. D. V. Widder, The Laplace Transform (Priceton University Press, 1946).

9. A. H. Zemanian, Some abelian theorems for the Distributional Hankel and $K$ transformations, SIAM J. Appl. Math., Vol. 14 (1966), 1106-1111.

Department OF Math.

CARLETON UNIVERSTTY,

OTtAWA, ONTARIO,

CANADA. 